\title{
Route Deviation Algorithm with Location Ambiguity in Wireless Sensor Networks
}

\author{
Priyanka Chugh (Shivanka), Dinesh Rai, S. Indu
}

\begin{abstract}
Nowadays, the primary concern of geographic routing protocol lies in the fact of minimisation of energy dissipation during the transfer of each packet in a network. This paper proposes an energy-efficient real-time algorithm in sensor networks, i.e., Route deviation Algorithm. Route deviation algorithm combines the characteristics of both distance-based criteria and direction or angle based criteria. In this paper, we have shown a comparison amongst COMPASS algorithm, NFP, $M E R$ and Route deviation algorithm. The simulation model includes numerous parameters, namely, threshold energy, number of sensors, spread of the map, position of sensors and transmission time. The results obtained through the simulation model supports the fact that the Route deviation algorithm accomplishes the task of saving energy and adds to the life of the networks.
\end{abstract}

Index Terms: Compass, NFP, MER, lifetime of network, wireless sensor network, Route deviation, transmission range.

\section{INTRODUCTION}

For forthcoming generations, wireless sensor networks are a significant technology that could be utilised for numerous purposes. The significant applications of Wireless sensor networks include military applications, obtrusion detection and environmental monitoring. A wireless sensor network consists of wireless sensor nodes which have low-puissance, are economically cheap, multifunctional, capable of sensing and computing, and can communicate wirelessly. These sensor nodes have been characterised based on restricted resources, specifically memory, energy and processing power [20]. These nodes mainly consist of data processing unit called CPU, a recollection unit for storing data, a transceiver for forwarding and receiving signals, data or packets and battery to provide energy for its functioning. Together, the nodes form a network enabling communication between these nodes. Nodes can communicate directly or via several intermediate nodes. The sending node becomes the source, which communicates with one or more nodes that act as sink. For a distant sink, a multi-hop protocol is utilised, i.e. the sink

Revised Manuscript Received on December 30, 2019.

* Correspondence Author

Priyanka Chugh (Shivanka), PhD Scholar, School of Engineering and Technology, Ansal University, New Delhi, India.

Dr Dinesh Rai, Associate Professor and Dean, School of Engineering and Technology, Ansal University, New Delhi, India.

Dr S. Indu, Professor in ECE Department, Delhi Technological University, New Delhi, India.

(c) The Authors. Published by Blue Eyes Intelligence Engineering and Sciences Publication (BEIESP). This is an open access article under the CC BY-NC-ND license (http://creativecommons.org/licenses/by-nc-nd/4.0/) node is off direct transmission range of the source node, the data is sent via chain of hops employing intermediate nodes. Since the resources are inhibited and the energy used is more, the routing process is cumbersome.

This work predicated on geographic routing as we commence by giving a brief exordium about identically tantamount. After that, it discusses its protocols, namely, Compass, NFP and MER. Proactive routing is a technique where topology of network discovered by broadcasting flare signal from the sink to the entire system periodically [19]. An extended incipient algorithm based route-deviation is proposed in this work. This algorithm predicts routes by identifying the node's routes with location errors taken into consideration. Here, those subsisting protocol's characteristics are analysed, which are helpful in more preponderant and energy-efficient based route establishment. Further, location errors are explored in detail to avoid energy losses. This work is organised as follows: an incipient algorithm based on route deviation with location errors is proposed in Section 2. This algorithm utilises the characteristics of both the subsisting protocols and results in a more dominant and energy-efficient routing of data packets. Section 3 discusses the errors that can occur due to faulty or approximated location coordinates and how our algorithm surmounts these errors, thereby incrementing the energy efficiency that is the significant difficulty nowadays. The simulation model in section 4 compares our algorithm with the subsisting protocols of geographic routing. Finally, Section 5 concludes this paper.

\section{SOME PRIOR DEVELOPMENTS}

The principle for routing the packets that is established utilising the geographic location of the node is termed as Geographic routing. Its application includes routing in wireless networks which fixates on the notion that the primary node sends a packet to the geographical location of the next node instead of routing the packet predicated on the network address of the node. The concept of utilising information about location for routing was first suggested in the field of packet radio networks during the 1980s [1] [10] [11] and interconnection networks. In Geographic routing, nodes can resolve their location. Also, the source is informed about the destination [2]. With this erudition of each node, the packet could be forwarded to the destination node without much information of the topology of the network or any earlier revelation of the route. Geographic routing includes several approaches like uni-path, multi-path and flooding-predicated strategies [3]. The uni-path procedure mainly consists of two techniques, namely, face routing [10] and greedy forwarding. Avaricious forwarding brings the packet proximate to the destination node utilising the spatial information of the node. 
Thus, each node sends the message packet to the most proximate node. The relevant neighbouring node is that which decreases the distance between the destination and source in every hop. Alternatively, another progressive approach is, namely estimated distance between source and destination (MFR, NFP), or the minimal angle that the neighbour makes with the destination node (Compass Routing). All these strategies are not looped free, i.e. a packet can infinitely move two and fro between nodes in some configuration. It has been optically discerned that the greedy strategy \& MFR protocol are liberated from loops, while Compass Routing and NFP are not.

The acquisitive forwarding variants are shown in Figure 1. The source (S) has several calls to find an intermediate node for forwarding the message further to the destination (D). Q = Nearest with Forwarding Progress (NFP), $\mathrm{R}=$ Most Forwarding progress within Radius (MFR), $\mathrm{P}=$ Compass Routing, $\mathrm{T}=$ Greedy.

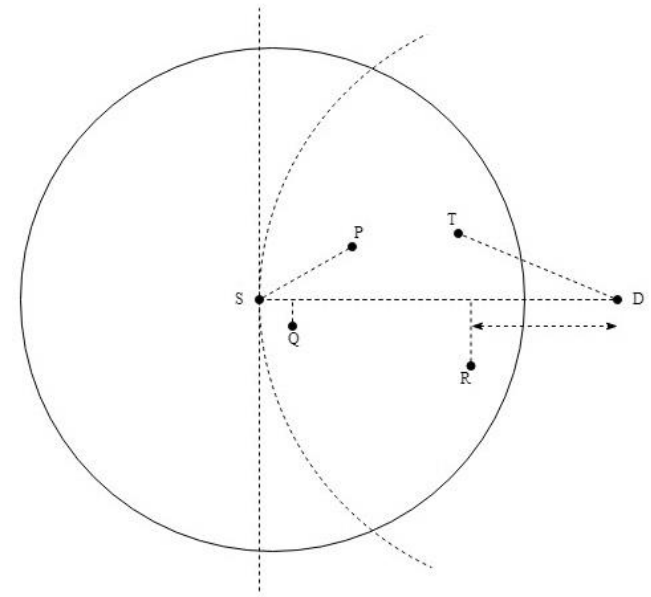

Figure 1: Greedy forwarding variants in Geographic Routing [3]

Face routing: Figure. 2 shows the face routing process in detail. A message packet, routed along the inside of faces of the graph of communication, where face changes at the edges intersecting the line P-Q.

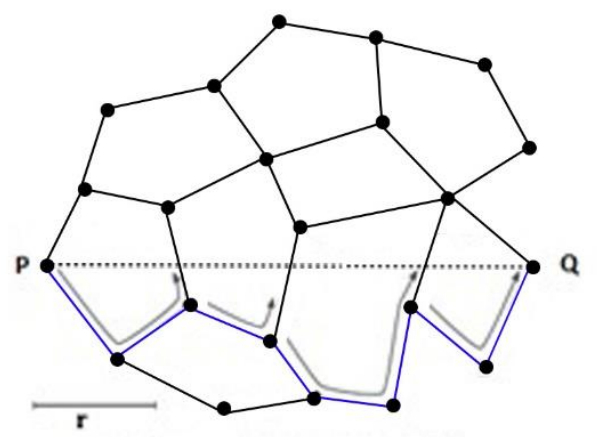

Figure 2: Face routing [4]

\section{A.Compass Routing}

If we opt to transmit a data packet, from starting point to endpoint, the detail available in the position of the node we are standing at currently, the location of the destination in terms of its coordinates and edges converging at the node we are standing at. Hence to reach the destination, we traverse along the edges incident on the node we are currently standing at having minimum slope with that of the destination node [4][6]. This minimises energy consumption. A detailed compass routing process is shown in Figure. 3.

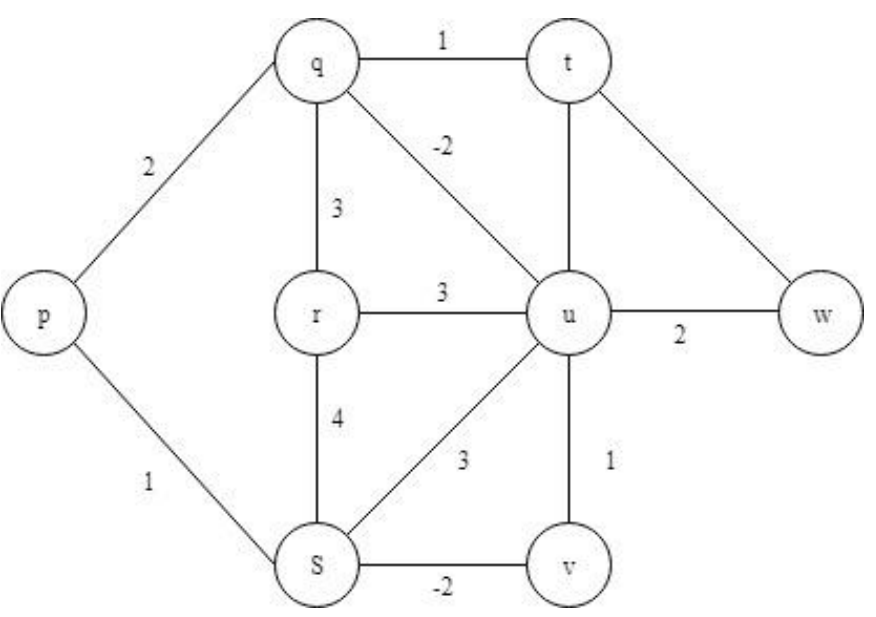

Figure 3: Compass Routing [6]

\section{B. Nearest with Forward Progress (NFP)}

NFP [5] is an energy-efficient protocol which minimises the energy consumption during packet transfer by sending the message to the most proximate node in the direction of the final destination node where the packet is destined to reach [6]. The NFP makes collisions less liable to occur as each node adjusts its transmission power to be just vigorous enough to reach the most proximate neighbour, which results in forward progress, which is also the main advantage of NFP. This leads to a large number of small hops resulting in high delivery latency and less energy consumption as compared to hops located at a considerable distance.

\section{Maximum Expectation within Transmission Range (MER)*}

MER is a geographic routing algorithmic rule that alleviates the impact of location errors on routing in wireless ad hoc networks. MER doesn't want predefined location information for operation. However, in practice, there could be remarkable errors in predicting location estimates. MER includes location errors into the main objective perform by considering both transmission failures and backward progress. Every one node then forwards packets to the node that maximises this actual output. MER is influential to the locality error model and model parameters but is not as effective in cases when the location of nodes is predefined.

\section{PROBLEM FORMULATION- PRESENCE OF SPATIAL ERRORS IN GEOGRAPHIC ROUTING}

Because of its simplicity in implementation and scalability, geographic routing is widely accepted as a major research challenge in wireless sensor networks.

In geographic routing, the nodes confirm their location (either by using the Global Positioning System (GPS) [11], [12] or alternative location-based positioning processes useful in wireless sensor networks [13]) and broadcast the data concerning location to alternative nodes consistently and proactively.

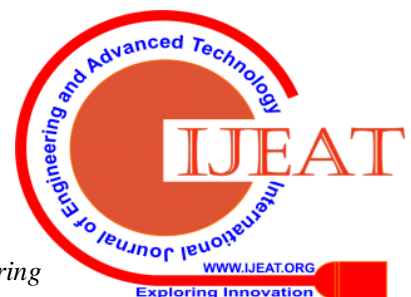


The transmitted packet is predicated using the

location of the neighbour that is stored in the database (DB) of each node and that of the destination is enclosed in the packet.

Thus GPS results depend [8] [11] on:

- Individual environment

- Geometry of satellite location-sensing technique

- Radio environment

In geographical routing, packet forwarding is done using the greedy method during which the nodes use essential information to send packets in the direction of their destination. The location error, Z, for a network is calculated by:

$$
Z=\frac{\overline{\mathrm{x}}-\mu}{\frac{\sigma}{\sqrt{\mathrm{N}}}}
$$

Where $\overline{\mathrm{x}}$ is the mean of nodes, $\mu$ is mean of population, $\sigma$ is standard deviation and $\mathrm{N}$, number of nodes in the wireless sensor network.

Here, the greedy node is observed for regular sessions. If it fails (i.e., the destination node unavailable, or forwarding results fails), in any of consecutive sessions then a recovery mode starts its job of finding a successful alternative route to its destination. In practice, location information that is received is not exact [11], [14], [15] and the performance and efficiency of geographic routing are degraded due to errors in information about the location of the nodes [15]. This incorrectness is caused even if the nodes utilise GPS because of the fundamental mistake of GPS in location evaluation [12]. The possible location errors are transmission range error and backward progress error. [8]

\section{A.Transmission range error}

The first location error in a particular place in geographic routing is posited, the area of transmission is chosen to be circular because each of the neighbouring code put other nodes in their range if this transmission phenomenon is followed. Usually, these phenomenon are followed when nodes lie within different transmission range. Further, these posit fails if nodes lie within other node's transmission range but generated transmission patterns have many irregular or faulty spaces [16]. Now, variable transmission range nodes may be present in the distributed wireless transmission region; thus, its presence within transmission range is not enough to be counted as part of a wireless sensor network. This link between a node and its neighbour is named as asymmetric link.

In associate degree asymmetric link, range failures during transmission will transpire in the presence of spatial inaccuracies even if every node exactly kens the transmission range as shown in Figure. 4. Hence, this location error needs to be eliminated by proposing a condition that each node must be within the scope of its neighbouring nodes before the packet is forwarded. In earlier experimental analysis, it is observed that Lost Link and Loop construction are two significant challenges. Thus, the neighbouring node's help is very much required for implementing the desired level of network construction and formulation. Loop construction can be avoided by tracing the devices on the path and leaving proper evidences

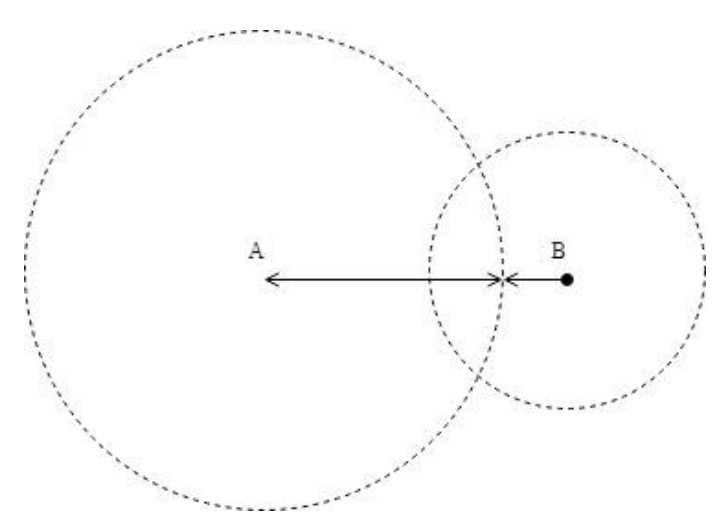

Figure 4: Irregular transmission pattern (scenario-1) [8]

\section{B. Backward Progress error}

This error occurs due to the placement of neighbouring nodes inside a network. A neighbouring node may be present far from destination as compared to its place with sending node as shown in Figure. 5. This could subsist a route when no destination's neighbouring node is helpful [8]. This is a challenge for local minimum solution in geographical routing based problem formulation and requires to active route recovery process [8]. This arises a loop problem between the sending node and its neighbouring nodes which are father from destination but closer to source. Hence, we propose a condition to avert the algorithm to enter this infinite loop to minimise the energy consumption during packet forwarding.

Figure 5: Irregular transmission pattern (Scenrio-2) [11]

\section{PROPOSED METHODOLOGY FOR ROUTE}

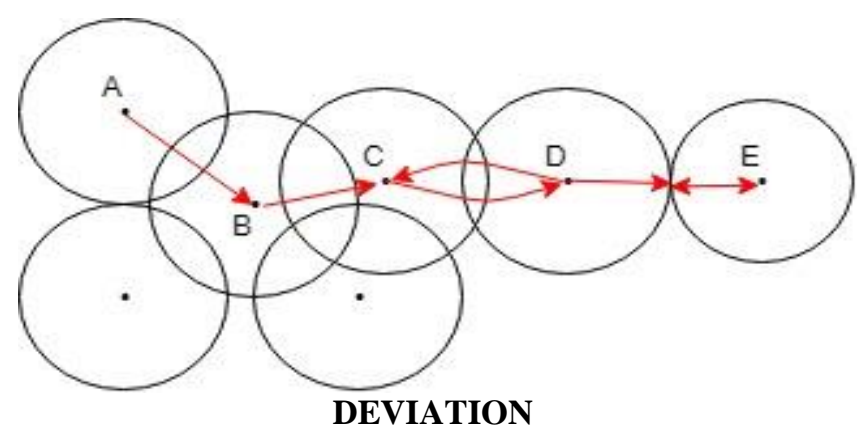

We propose the algorithm that utilises the characteristics of both the NFP and the compass protocol, as shown in Figure. 6. The hybrid is termed as Route deviation Protocol with location errors. The algorithm takes into account all the necessary conditions' check that may cause an error due to location coordinates or battery issues.

\section{A. Algorithm}

\section{1) Assumptions}

Let there be a node $\mathrm{N}$ which has received a packet. The node $N$ has neighbouring nodes, verbally express, n[i] where i is the number of immediate neighbours of node $\mathrm{N}$. The angle that each neighbour, $n[i]$ makes with the line joining the source and destination is $\theta . n[i]$. The distance of each neighbour is $Đ . n[\mathrm{i}]$.

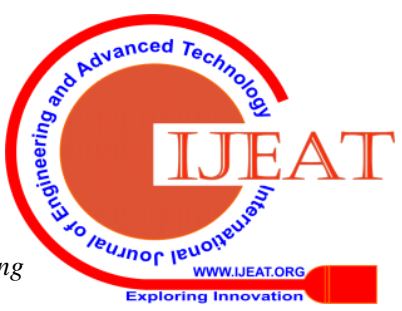




\section{Route Deviation Algorithm with Location Ambiguity in Wireless Sensor Networks}

2) The Route deviation Factor

The factor $\mathrm{S}$ of route deviation algorithm is calculated utilising:

$$
\mathrm{S}=\text { distance / angle i.e., } \mathrm{S}=\mathrm{Ðn}[\mathrm{i}] / \theta \mathrm{n}[\mathrm{i}] \text {. }
$$

\section{3) Initial Conditions}

After receiving the packet to any of the network nodes, the battery of that node is checked. If the battery is low, the destination becomes unreachable and the algorithm exits. The node is additionally checked if it is the destination node. If true, then withal the algorithm exits. The route deviation factor, $S$, is initialised to zero.

\section{4) Check Location Errors}

The next node $n[i]$ is checked for two possible errors that can occur due to location coordinates:

a) Backtracking (looping): The node $n[i]$ is checked if it is not one of the parent nodes, i.e., the route should not be in a backward direction.

b) Transmission Range: Each node afore forwarding the packet should check if the next node lies within its range and the sending node is in the range of the next node.

\section{5) Distance comparison}

Afore calculating the factor $\mathrm{S}$ for each neighbour, the distance from source for each neighbour is checked. If any neighbour whose distance from source is minuter as compared to the distance from the source of node $\mathrm{N}$, it is neglected.

\section{6) Computation}

- For neighbours that are in the forward direction, i.e., those that are proximate to the destination, the factor $S$ is calculated.

- The factor $\mathrm{S}$ for each neighbour is compared amongst each other. The neighbour which has maximum value for $\mathrm{P}$ is the next node to which packet is transferred.

- Then again, the above steps are followed for the next node until the destination is reached or it becomes unreachable because of the low power of the battery.

\section{B. Route Deviation Algorithm}

Variables: Nodes n, number of nodes $\mathrm{i}, \mathrm{i}^{\text {th }}$ neighbouring node $\mathrm{n}[\mathrm{i}]$, distance of $\mathrm{n}[\mathrm{i}]$ node $\mathrm{Ð}_{\mathrm{n}[\mathrm{i}}$, angle between node $\mathrm{n}[\mathrm{i}]$ and

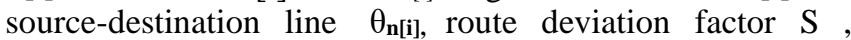
Destination node $n_{d}$, Parent node of node $n[i]$ is $n_{p}[i]$, node to which the packet is forwarded. Assuming the packet is received, the detailed proposed route deviation algorithm is discussed in algorithm 1.

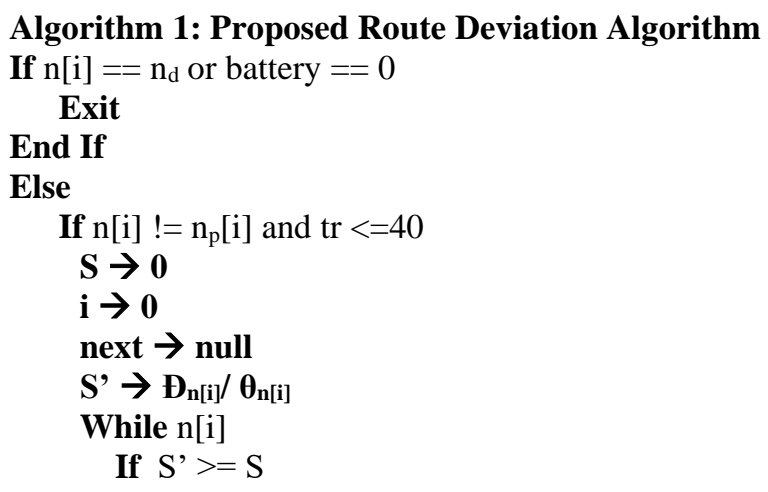

\section{End If \\ Else}

EXIT

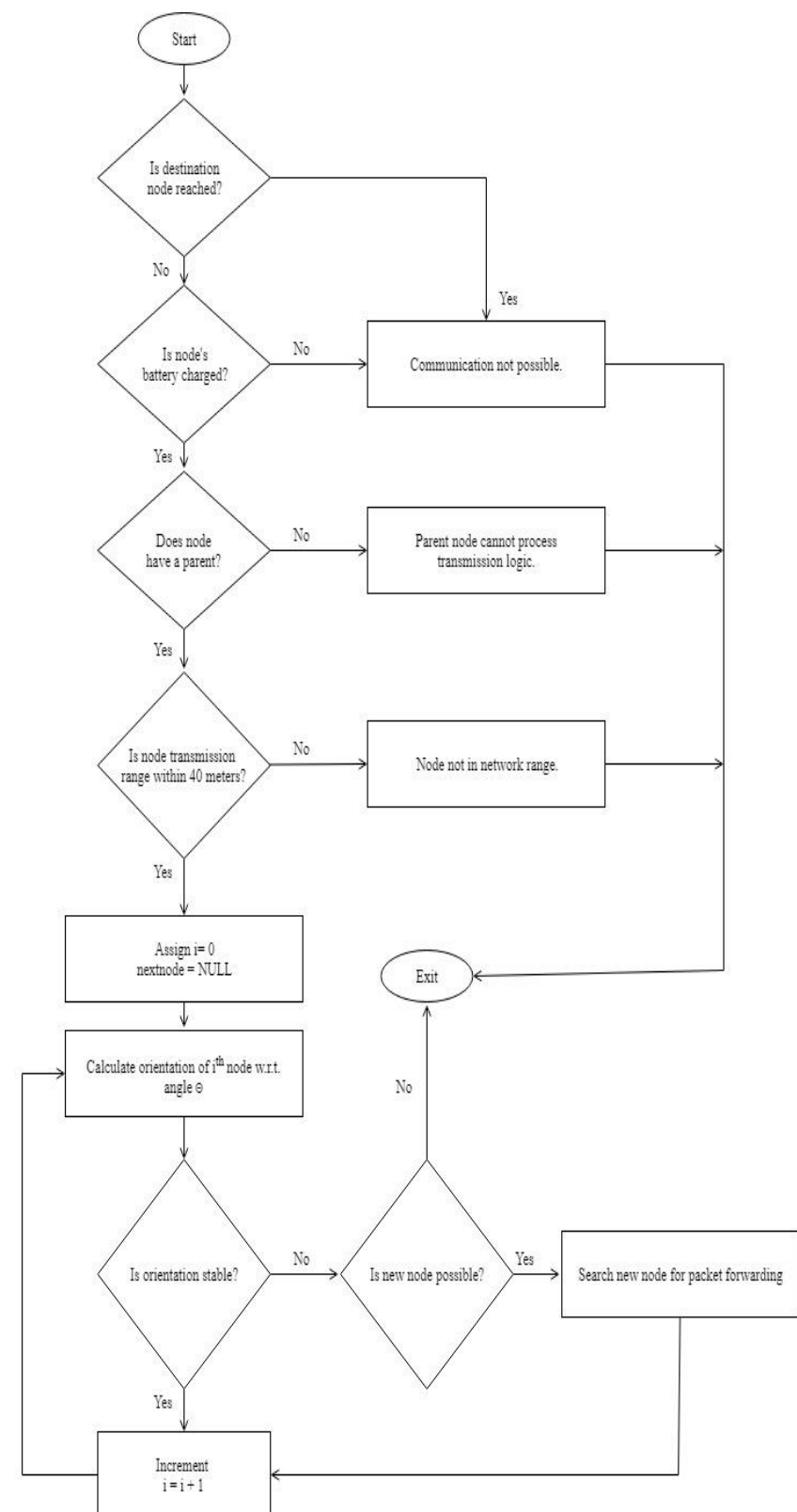

Figure 6: Flowchart for Route deviation Algorithm with Location Errors

\section{SIMULATION AND ANALYSIS}

In simulation proposed algorithm is compared with NFP and compass algorithm utilising the simulation model. Various simulation parameters taken for analysis are shown in Table 1. The simulation of the lifetime of WSN is performed by employing a simulator developed using JAVA programming language and OOP development paradigm. This simulator takes the parameters given below. The values of all the parameters as shown in Table 1 are provided by default and can withal be transmuted to provide custom values for simulation. 
Table 1: Default values of parameters

\begin{tabular}{|l|l|}
\hline Parameters & Initial Values \\
\hline Sink X location & 0 \\
\hline Sink Y location & 0 \\
\hline Knowledge Range & 30.0 \\
\hline Initial Battery Capacity & 10000 \\
\hline Overhead Energy & 0.02 \\
\hline Transmission Time & 250 \\
\hline Packet Arrival Rate & 60 \\
\hline Path Loss Exponent & 3.0 \\
\hline Lifetime Threshold & 0.60 \\
\hline Sensor Number & 200 \\
\hline Map Dimension & 200 \\
\hline
\end{tabular}

\section{A. Default Parameters}

The parameters used are:

- Location of sink node in $\mathrm{X}$ and $\mathrm{Y}$ coordinates

- Maximum Range of Transmission of each node

- Overhead Energy

- Total energy of the system

- Time duration for which Transmission takes place

- Rate of Arrival of Packets

- Path loss exponent

- Lifetime Threshold

- Total Number of Sensors

- Dimensions of map

\section{Network Lifetime and Reliability}

Network lifetime has turned into a fundamental characteristic to evaluate the sensor networks in an application inhibited way. The analysis of network lifetime includes the information about available nodes, the coverage area of the sensor, and the connectivity. The quality of accommodation can be quantified in terms of life considerations. Number of methods and algorithmic processes are suggested to increment the simulation time of wireless sensor networks while the evaluations of the networks were always predicated on a concrete definition of network lifetime. Network lifetime is defined as: "Proportion of available power $(\mathrm{Pa})$ to total potency $(\mathrm{Pt})$, proportion of live sensors to total sensors, and proportion of live sink sensors to total sink sensor."

\section{Threshold Energy}

Threshold Energy is proportionate to the distance of a node from its base station. If a node has to transmit a packet to any other node inside a network, then a threshold energy is required to send a packet from its location to base station. The energy threshold is the deciding factor for communication with the base station. This is the minimum energy required from every active node in the network such that nodes can communicate with other nodes in the presence of a base station.

\section{Transmission Range}

Erudition or Transmission range is a coverage area within which nodes can communicate with a base station or other node.

\section{Overhead Energy}

The initial energy of a node is kenned as overhead energy.

\section{B.Scenarios:}

The following cases were analysed; each case varies a particular parameter while other parameters are kept constant. A graph exhibiting the relationship between overhead energy and network lifetime as well as reliability is additionally shown for each case.

Scenario 1:

By keeping the transmission time to $250 \mathrm{~ms}$ and varying the value of overhead energy $\left(E_{0}\right)$, we observed the values of reliabilities of NFP, COMPASS, ROUTE DEVIATION and MER as shown in Table 2. A corresponding graph between $\mathrm{E}_{0}$ and Reliability is shown in Figure. 7.

Table 2: E0 vs Reliability when transmission time is $250 \mathrm{~ms}$

\begin{tabular}{|c|c|c|c|c|}
\hline \multirow{2}{*}{ E0 } & \multicolumn{4}{|c|}{ RELIABILITY } \\
\cline { 2 - 5 } & $\begin{array}{c}\text { ROUTE } \\
\text { DEVIATION }\end{array}$ & MER & NFP & COMPASS \\
\hline 0.1 & 0.51 & 0.49 & 0.35 & 0.4 \\
\hline 0.25 & 0.555 & 0.54 & 0.47 & 0.53 \\
\hline 0.55 & 0.58 & 0.56 & 0.525 & 0.545 \\
\hline 0.75 & 0.585 & 0.58 & 0.54 & 0.555 \\
\hline 0.95 & 0.595 & 0.585 & 0.565 & 0.575 \\
\hline
\end{tabular}

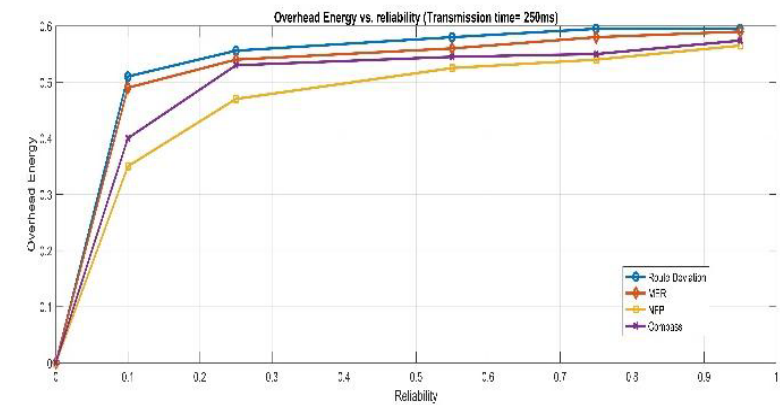

Figure 7: Overhead Energy vs reliability (Transmission time $=250 \mathrm{~ms}$ )

Route Deviation Algorithm performs better than NFP and Compass but with a slight increase in overhead energy. 


\section{Route Deviation Algorithm with Location Ambiguity in Wireless Sensor Networks}

Scenario 2:

In this case, the transmission time is kept at $750 \mathrm{~ms}$, and for different values of Eo, we observe the values of reliabilities of NFP, COMPASS, ROUTE DEVIATION and MER as shown in Table 3. A corresponding graph between Eo and Reliability is shown in Figure 8.

Table 3. E0 vs Reliability when Transmission time is $750 \mathrm{~ms}$

\begin{tabular}{|c|c|c|c|c|}
\hline \multicolumn{2}{|c|}{ E0 } & \multicolumn{3}{c|}{ RELIABILITY } \\
\hline ROUTE DEVIATION & MER & \multicolumn{2}{|c|}{ NFP } & COMPASS \\
\hline 0.10 & 0.290 & 0.270 & 0.250 & 0.260 \\
\hline 0.20 & 0.480 & 0.460 & 0.370 & 0.410 \\
\hline 0.55 & 0.560 & 0.520 & 0.4747 & 0.490 \\
\hline 0.75 & 0.595 & 0.570 & 0.500 & 0.530 \\
\hline 0.90 & 0.595 & 0.590 & 0.525 & 0.535 \\
\hline
\end{tabular}

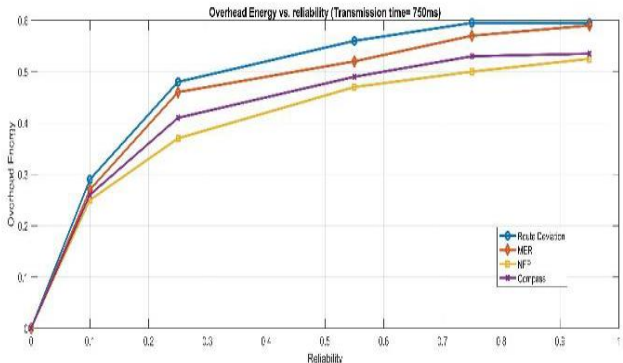

Figure 8: Overhead Energy vs reliability (Transmission time $=750 \mathrm{~ms}$ )

Scenario 3:

In this case, for transmission time 250ms and threshold energy 0.6J. The observation table for this case is shown in Table 4, and the corresponding graph is shown in Figure 9.

Table 4: Overhead energy vs Lifetime when Threshold energy is $0.60 \mathrm{~J}$

\begin{tabular}{|c|c|c|c|c|}
\hline \multicolumn{3}{|c|}{ OVERHEAD ENERGY } & \multicolumn{2}{|c|}{ LIFETIME } \\
\hline \multicolumn{2}{|c|}{ ROUTE DEVIATION } & \multirow{2}{*}{$\begin{array}{r}\text { MER } \\
383 \\
\end{array}$} & \multirow{2}{*}{$\begin{array}{l}\text { NFP } \\
324 \\
\end{array}$} & \multirow{2}{*}{$\begin{array}{c}\text { COMPASS } \\
360 \\
\end{array}$} \\
\hline 0.02 & 396 & & & \\
\hline 0.10 & 412 & 399 & 375 & 385 \\
\hline 0.15 & 401 & 387 & 379 & 386 \\
\hline 0.20 & 387 & 375 & 382 & 362 \\
\hline 0.25 & 410 & 396 & 355 & 380 \\
\hline
\end{tabular}

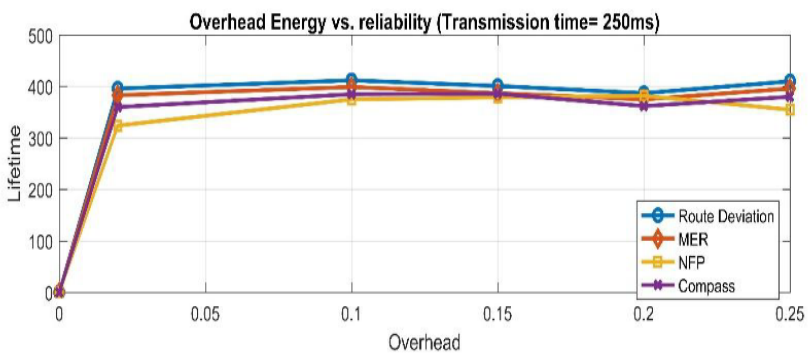

Figure 9: Overhead Energy vs Lifetime (Transmission time $=250 \mathrm{~ms}$ )

\section{Scenario 4:}

This scenario shows variation in reliabilities for different transmission ranges. With the increase in transmission range beyond 40 , there is a steep decrease in reliability exhibiting the existence of spatial errors. The cumulated effect of transmission range error and backward progress error is optically discerned in this case. The observations are shown in Table 5 and corresponding graph in Figure 10.

Table 5: Transmission range vs reliability when network range is $\mathbf{4 0 .}$

\begin{tabular}{|c|c|c|c|c|}
\hline \multicolumn{3}{|c|}{ TRANS } & \multicolumn{2}{c|}{ RELIABILITY } \\
\hline MISSION RANGE & \multicolumn{2}{c|}{} \\
\hline 37 & 0.33 & 0.31 & 0.29 & 0.30 \\
\hline 39 & 0.52 & 0.49 & 0.46 & 0.50 \\
\hline 42 & 0.53 & 0.48 & 0.57 & 0.44 \\
\hline 46 & 0.23 & 0.26 & 0.43 & 0.52 \\
\hline 50 & 0.32 & 0.29 & 0.32 & 0.34 \\
\hline
\end{tabular}

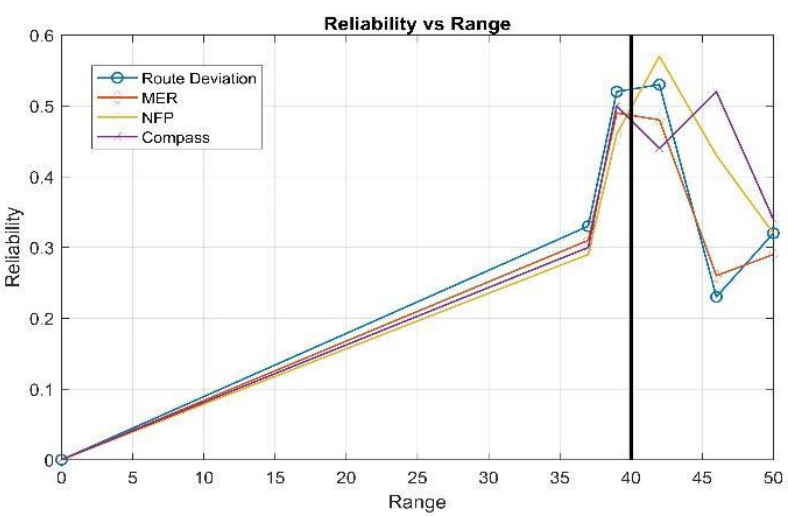

Figure 10: Reliability vs Range

Scenario 5:

This scenario shows the variation in reliability (no of packets received) and lifetime for different Threshold Lifetime. It is observed from the graph that with the increase in threshold lifetime, Reliability and System Lifetime increases linearly which is the desired feature of a wireless sensor network. Table 6 and Table 7 display this observation for two sinks $\mathrm{X}$ and $\mathrm{Y}$. The corresponding graphs are drawn in Figure. 11,12 and 13.

Table 6: Variation of Reliability with respect to Threshold Lifetime

\begin{tabular}{|c|c|c|c|}
\hline SINK X & SINK Y & $\begin{array}{c}\text { Threshold } \\
\text { Lifetime }\end{array}$ & Reliability \\
\hline 0 & \multirow{2}{*}{0} & 0.40 & 0.37 \\
\cline { 3 - 4 } & & 0.50 & 0.38 \\
\cline { 3 - 4 } & & 0.60 & 0.59 \\
\cline { 3 - 4 } & & 0.70 & 0.68 \\
\cline { 3 - 4 } & & 0.80 & 0.73 \\
\cline { 3 - 4 } & & 0.90 & 0.78 \\
\cline { 3 - 4 } & \multirow{4}{*}{25} & 1 & 0.93 \\
\cline { 3 - 4 } & & 0.40 & 0.39 \\
\cline { 3 - 4 } & & 0.50 & 0.48 \\
\cline { 3 - 4 } & & 0.60 & 0.64 \\
\cline { 3 - 4 } & & 0.70 & 0.73 \\
\cline { 3 - 4 } & & 0.80 & 0.78 \\
\hline
\end{tabular}




\begin{tabular}{|c|c|c|c|}
\hline & & 0.90 & 0.83 \\
\hline & & 1 & 0.96 \\
\hline \multirow[t]{7}{*}{50} & \multirow[t]{7}{*}{50} & 0.40 & 0.45 \\
\hline & & 0.50 & 0.57 \\
\hline & & 0.60 & 0.68 \\
\hline & & 0.70 & 0.78 \\
\hline & & 0.80 & 0.83 \\
\hline & & 0.90 & 0.88 \\
\hline & & 1 & 0.99 \\
\hline \multirow[t]{7}{*}{100} & \multirow[t]{7}{*}{100} & 0.40 & 0.62 \\
\hline & & 0.50 & 0.65 \\
\hline & & 0.60 & 0.73 \\
\hline & & 0.70 & 0.83 \\
\hline & & 0.80 & 0.85 \\
\hline & & 0.90 & 0.90 \\
\hline & & 1 & 0.98 \\
\hline
\end{tabular}

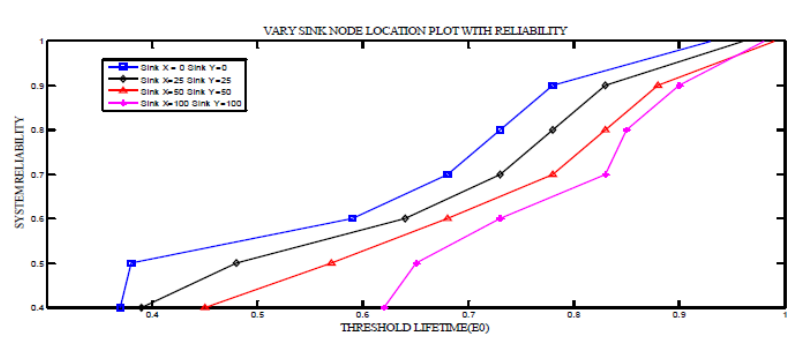

Figure 11: Variation of System Reliability with Threshold Lifetime for different Sink Node Location

Table 7: Variation of System Lifetime with respect to Threshold Lifetime

\begin{tabular}{|c|c|c|c|}
\hline 0 & 0 & 0.40 & 3141000 \\
\hline \multicolumn{2}{|c|}{0.50} & \multicolumn{2}{|c|}{3242000} \\
\hline \multicolumn{2}{|c|}{0.60} & \multicolumn{2}{|c|}{3401000} \\
\hline \multicolumn{2}{|c|}{0.70} & \multicolumn{2}{|c|}{3500750} \\
\hline \multicolumn{2}{|c|}{0.80} & \multicolumn{2}{|c|}{3520756} \\
\hline \multicolumn{2}{|c|}{0.90} & \multicolumn{2}{|c|}{2870210} \\
\hline \multicolumn{2}{|c|}{1} & \multicolumn{2}{|c|}{0} \\
\hline 25 & 25 & 0.40 & 3243432 \\
\hline \multicolumn{2}{|c|}{0.50} & \multicolumn{2}{|c|}{3381000} \\
\hline \multicolumn{2}{|c|}{0.60} & \multicolumn{2}{|c|}{3581000} \\
\hline \multicolumn{2}{|c|}{0.70} & \multicolumn{2}{|c|}{3600500} \\
\hline \multicolumn{2}{|c|}{0.80} & \multicolumn{2}{|c|}{3621500} \\
\hline \multicolumn{2}{|c|}{0.90} & \multicolumn{2}{|c|}{3200500} \\
\hline \multicolumn{2}{|c|}{1} & \multicolumn{2}{|c|}{0} \\
\hline 50 & 50 & 0.40 & 3301250 \\
\hline \multicolumn{2}{|c|}{0.50} & \multicolumn{2}{|c|}{3481000} \\
\hline \multicolumn{2}{|c|}{0.60} & \multicolumn{2}{|c|}{3680500} \\
\hline \multicolumn{2}{|c|}{0.70} & \multicolumn{2}{|c|}{3700750} \\
\hline \multicolumn{2}{|c|}{0.80} & \multicolumn{2}{|c|}{3784750} \\
\hline \multicolumn{2}{|c|}{0.90} & \multicolumn{2}{|c|}{3600250} \\
\hline \multicolumn{2}{|c|}{1} & & 0 \\
\hline 100 & 100 & 0.40 & 3462000 \\
\hline & & & 2500 \\
\hline & & & 2500 \\
\hline
\end{tabular}

\begin{tabular}{|c|c|}
\hline 0.70 & 3807500 \\
\hline 0.80 & 3880500 \\
\hline 0.90 & 3800000 \\
\hline 1 & 0 \\
\hline
\end{tabular}

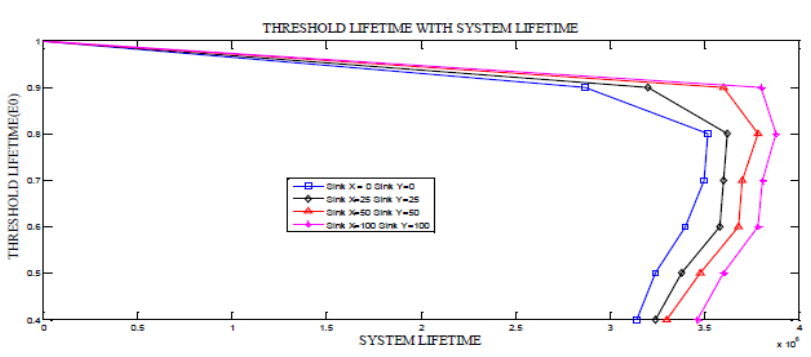

Figure 12: Variation of System Lifetime with Threshold Lifetime for different Sink Location

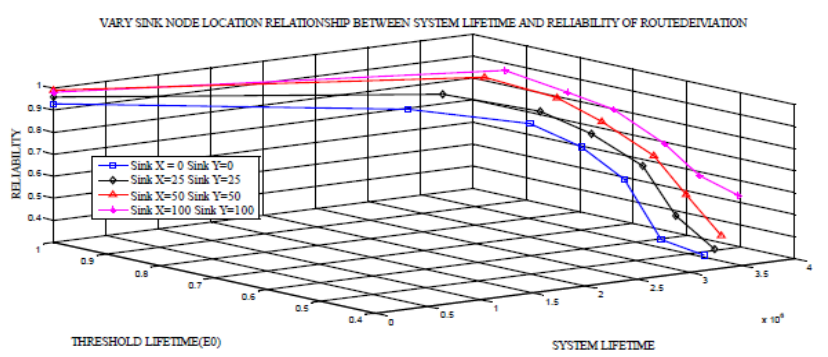

Figure 13: A 3-dimensional graph of System Lifetime, Threshold Lifetime and Reliability for different Sink Location.

\section{Protocol Achievement}

As we analysed ROUTE DEVIATION algorithm for reliability and lifetime of WSN by comparing it with different protocols and varying various parameters utilised in simulation, we optically discerned that reliability of WSN is scarcely affected by ROUTE DEVIATION protocol and the lifetime of the WSN is enhanced to quite an extent in comparison to NFP and COMPASS.

The analysis shows that route deviation gives an optimum result as compared to MER, NFP and Compass. As we can visually perceive from the graphs that when overhead energy is 0.3 and threshold is $0.60 \mathrm{~J}$, the ROUTE DEVIATION protocol gives us the optimum lifetime of 420, whereas MER gives the value of 412, COMPASS algorithm gives the value of 402 and NFP 390. The reliability observed for the same case is 0.565 for Route deviation whereas 0.56 for MER, 0.55 for Compass and 0.52 for NFP.

With existing routing criteria [Hou \& Li 1986] [Shivanka et al. 2013], following are the observations:

- In Table 2, the reliability of ROUTE DEVIATION is approximately $1.7 \%$ more preponderant than COMPASS and $14.2 \%$ more preponderant than NFP.

- In Table 3, the reliability of ROUTE DEVIATION is approximately $1.78 \%$ more preponderant than COMPASS and $21.4 \%$ more preponderant than NFP.

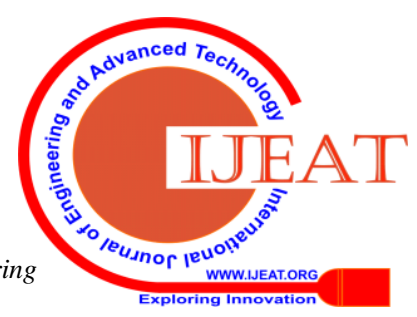


- In Table 4, the lifetime of ROUTE DEVIATION is approximately $52.98 \%$ more preponderant than COMPASS and $44.8 \%$ more preponderant than NFP.

- Table 5 shows vicissitudes in reliability within the transmission range and beyond the range. The Route deviation algorithm shows a $58.9 \%$ increase in reliability after the implementation of the solution of the location errors discussed in section III while Compass and NFP show a decrement in reliabilities by $5.4 \%$ and $6.3 \%$ respectively.

\section{CONCLUSION}

We transmuted the Transmission Time and the Threshold Energy to evaluate the lifetime and reliability of the network that availed us to prosperously find a route to the destination node in each routing protocol. Moreover, when we defined the condition of transmission range, we observed that reliability decremented after a range of the network designated, which minimised the energy dissipation. When culling a different transmission time of nodes, we test our proposed routing protocol ROUTE DEVIATION performs preponderant in comparison to Compass and NFP in case of reliability. When culling Threshold Energy at different values, and finding overhead energy vs reliability and lifetime, we optically discern that our protocol performs significantly preponderant than the Compass and NFP and enhances the overall performance of the network.

\section{ACKNOWLEDGMENT}

The authors would like to thank and acknowledge anonymous reviewers for their valuable consecutive comments to improve the quality of this paper.

\section{REFERENCES}

1. Takagi.H., L.Kleinrock, 1984. Optimal transmission ranges for randomly distributed packet radio terminals. IEEE Transactions on Communications 32 (3) 246-257 [doi:10.1109/TCOM.1984.1096061].

2. Finn, Gregory G March 1987. Routing and Addressing Problems in Large Metropolitan-Scale Internetworks. University of Southern California, ISI/RR-87-180.

3. Stojmenovic, Ivan, 2002. Position based routing in ad hoc networks. IEEE $\begin{array}{lllll}\text { Communications } & \text { Magazine } & 40 & \text { (7): } & \text { 128-134 }\end{array}$ [doi:10.1109/MCOM.2002.1018018].

4. Evangelos Kranakis, Harvinder Singh, and Jorge Urrutia August 1999. Compass routing on geometric networks. Proc. 11th Canadian Conference on Computational Geometry, pp. 51-54.

5. T.-C. Hou and V. Li, 1986. Transmission range control in multihop packet radio networks. IEEE Transactions on Communications, 34:3844.

6. Shivanka, Gourav Chadha, Karan Naveen, Ashwani Kumar, March 2013 (Special Issue). Performance Analysis of Wireless Sensor Networks Using Compass and Nearest with Forward Progress Algorithms", Volume 4, No. 3, International Journal of Advanced Research in Computer Science.

7. J. Zhao and R. Govindan, 2003.Understanding packet delivery performance in dense wireless sensor networks. Proceedings of the First International Conference on Embedded Network Sensor Systems.page 1 - 13 [doi $>10.1145 / 958491.958493]$

8. Sungoh Kwon, Ness B. Shroff, October 2006. Geographic routing in the presence of location errors, Computer Networks: The International Journal of Computer and Telecommunications Networking, v.50 n.15,p.2902-2917

9. F. Kuhn, R. Wattenhofer, and A. Zollinger, 2003. Worst-case optimal and average-case efficient geometric ad-hoc routing in ACM MobiHoc, pp267-278

10. S. Ratnasamy, B. Karp, L. Yin, F. Yu, D. Estrin, R. Govindan, and S. Shenker, 2002. GHT-a geographic hash table for data-centric storage, in First ACM International Workshop on Wireless Sensor Networks and their Applications, pp. 78-87.

11. P. Misra, B. P. Burke, and M. M. Pratt, 1999.GPS performance in navigation, Proceedings of the IEEE, vol. 87, no. 1, pp. 65-85.

12. P.Misra and P. Enge, 2001.Global Positioning System: Signals, Measurements, and Performance. Massachusetts: Ganga-Jamuna Press.
13. J. Hightower and G. Borriello, August 2001. Location systems for ubiquitous computing, Computer, vol. 34, no. 8, pp. 57-66.

14. S. Slijepcevic, S. Megerian, and M. Potkonjak, 2002.Location errors in wireless embedded sensor networks: Sources, models, and effects on applications, ACM SIGMOBILE Mobile Computing and Communications Review, vol. 6, no. 3, pp. 67-78.

15. Y. Kim, J. Lee, and A. Helmy, 2003. Impact of location inconsistencies on geographic routing in wireless networks, in ACM International Workshop on Modeling, Analysis and Simulation of Wireless and Mobile Systems (MSWiM), pp. 124-127.

16. T. He, C. Huang, B. M. Blum, J. A. Stankovic, and T. Abdelzaher, 2003. Range-free localisation schemes for large scale sensor network, in ACM MobiCom, pp. 81-95.1735-1746

17. I.F. Akyildiz, W. Su, Y. Sankarasubramaniam, E. Cayirci, A survey on sensor networks, IEEE Communications Magazine 40 (2002) 102-114

18. A.A. Nezhad, D. Makrakis, A. Miri, Anonymous topology discovery for multihop wireless sensor networks, in Proceedings of 3rd ACM Workshop on QoS and Security For Wireless and Mobile Networks, Q2SWinet '07, Chania, Crete

19. M.I.Khan, W.N.Gansterer, G.Haring, Static vs Mobile Sink: The influence of basic parameter on energy efficiency in wireless sensor networks, In International Journal of computer communications vol. 36,issue 9,pp.965-978

20. I.F. Akyildiz, W. Su, Y. Sankarasubramaniam, E. Cayirci A survey on sensor networks, IEEE Communications Magazine, 40 (2002), pp. 102-114.

\section{AUTHORS PROFILE}

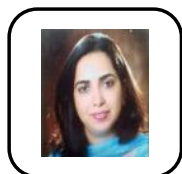

Ms Priyanka Chugh (Shivanka), received he M.Tech(CSE) from Mullana University Ambala in 2010, and she has completed B.Tech(IT) from Kurukshetra University in 2005. She has worked as Assistant Professor in Maharaja Surajmal Institute of Technology for 12 years (July 2007 to July 2019) in Information Technology department affiliated from GGSIPU New Delhi, and She has also worked as Lecturer in M. M.E.C Mullana University for six months as well as lecturer in C.I.T.M college Faridabad for six months. She has received a letter of appreciation from SMEs for excellent result in 2016 along with it she won $3^{\text {rd }}$ prize for supervising the project for B.Tech Intercollege project competition in Nov 2013. Her research area in Wireless Sensor Network and she has published three research paper International reputed journal and three research paper published in the International conference and one book chapter in Springer.

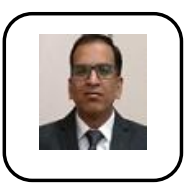

Dr Dinesh Rai, received his PhD (CSE) area Network security in 2013 from MONAD University as well as he has completed his M.tech (CSE) from National Institute of Technology Raipur in 2003 along with it He has completed his M.Sc degree in 1998 from Jiwaji University Gwalior. $\mathrm{He}$ is associated with Ansal University for more than twelve years. He is presently working as Associate Professor with designation Dean in Ansal University for School of Engineering and Technology for Last 12 years. Prior to joining Ansal University, he worked as Asstt. Prof. at Dronachraya College of Engineering, Gurgaon and GLA Institute of Technology, Mathura as Lecturer and Senior Lecturer. Dr Rai has published his research work in a good journal and presented some papers in international conferences. His current research interest is in the area of Cloud computing and Data sciences. Two of the projects done by him are still being used. One (named Quarterly Tax Plan System) at Transport Commissioner Office, M.P. and Other in KR Govt. Hospital, Gwalior. He Guided students of B.Tech (CSE) (now Alumni) on project 'E-Gas Sewa' to participate and won prize in IBM contest "The Great Mind Challenge." He has also got Best Innovative practitioner award by Wipro mission10x. He was chairperson in a technical session titled "Security issues in implementing Smart cities" in International Conference on Smart cities at Ansal University. 
Dr S. Indu, received her $\mathrm{PhD}$ (ECE) in 2012 from Delhi University as well as she completed her M.Tech (Control System) in 1990 from University of Kerala along with it She has completed her Bachelor of Engineering(Electrical Engineering) degree in 1987 from University of Kerala. She has many achievements in her life like She was University topper for M.Tech and Branch Counselor - IEEE DTU Student Branch Aug 2012-Aug 2017 as well as Best Branch Counselor award 2014 IEEE USA and Outstanding Branch Counselor Award for consecutive 5 years, IEEE Delhi Section along with it Best paper award ICCI-SEM-2017 (Computer session) and she has received a Commendable research award 2018 and Worked as General Chair ICSPVCE 2019. Currently, she is working as a professor in the ECE Department of Delhi Technological University. She was former head of the ECE Department. Now she holds the position of DEAN (Student Welfare). 